\title{
Insertional mutagenesis screening identifies the zinc finger homeodomain 2 (zfh2) gene as a novel factor required for embryonic leg development in Tribolium castaneum
}

\author{
Maike Kittelmann • Johannes B. Schinko • \\ Marco Winkler • Gregor Bucher • Ernst A. Wimmer • \\ Nikola-Michael Prpic
}

Received: 27 May 2009 / Accepted: 30 August 2009/Published online: 17 September 2009

(C) The Author(s) 2009. This article is published with open access at Springerlink.com

\begin{abstract}
The genetic control of leg development is well characterized in the fly Drosophila melanogaster. These control mechanisms, however, must differ to some degree between different insect species to account for the morphological diversity of thoracic legs in the insects. The legs of the flour beetle Tribolium castaneum differ from the Drosophila legs in their developmental mode as well as in their specific morphology especially at the larval stage. In order to identify genes involved in the morphogenesis of the Tribolium larval legs, we have analyzed EGFP enhancer trap lines of Tribolium. We have identified the $z f h 2$ gene as a novel factor required for normal leg development in Tribolium. RNA interference with $z f h 2$ function leads to two alternative classes of leg phenotype. The loss of a leg segment boundary and the generation of ectopic outgrowths in one class of phenotype suggest a role in leg segmentation and segment growth. The malformation of the pretarsal claw in the second class of phenotype suggests a role in distal development and the morphogenesis of the claw-
\end{abstract}

Communicated by S. Roth

M. Kittelmann · J. B. Schinko $\cdot$ M. Winkler $\cdot$ G. Bucher $\cdot$

E. A. Wimmer $\cdot$ N.-M. Prpic $(\varangle)$

Johann-Friedrich-Blumenbach-Institut für Zoologie

und Anthropologie, Abteilung Entwicklungsbiologie, GZMB,

Ernst-Caspari-Haus, Georg-August-Universität,

Justus-von-Liebig-Weg 11,

37077 Göttingen, Germany

e-mail: nprpic@uni-goettingen.de

M. Kittelmann

Center for Molecular Physiology of the Brain (CMPB),

European Neuroscience Institute,

Grisebachstraße 5,

37077 Göttingen, Germany shaped morphology of the pretarsus. This suggests that $z f h 2$ is involved in the regulation of an unidentified target gene in a concentration-dependent manner. Our results demonstrate that enhancer trap screens in $T$. castaneum have the potential to identify novel gene functions regulating specific developmental processes.

Keywords Tribolium castaneum · Leg morphogenesis . Appendage diversity · Genetic control · Insect morphology

\section{Introduction}

The genetic control of leg development has been studied in some detail in the fly Drosophila melanogaster. The primordia of the legs are specified in the Drosophila embryo by Wingless (Wg) signaling (e.g., Cohen et al. 1993). The leg-promoting function of $\mathrm{Wg}$ is counteracted on the dorsal side by Decapentaplegic (Dpp) signaling and on the ventral side by Egfr signaling, leading to the specification of the leg primordia on the ventral-lateral side of the body (Kubota et al. 2000, 2003; Goto and Hayashi 1997). The development of the proximal-distal axis of the legs is orchestrated by Wg signaling together with Dpp signaling. These two signaling pathways activate several target genes [e.g., Distal-less (Dll), dachshund (dac)] in a concentrationdependent manner (e.g., Lecuit and Cohen 1997). These target genes pattern the leg along its proximal-distal axis and coordinate the formation of the leg segment boundaries and the growth of the leg segments (podomeres) by instructing the Notch signaling pathway to be active at the future leg joints (Rauskolb and Irvine, 1999).

Many aspects of the genetic control of leg development in Drosophila are conserved in other arthropod species. 
For example, the expression and function of the Dll gene (e.g. Abzhanov and Kaufman 2000; Prpic et al. 2003; Schoppmeier and Damen 2001; Beermann et al. 2001), and the control of leg segmentation and podomere growth by Notch signaling (Prpic and Damen 2009) are highly conserved. On the other hand, the morphology of arthropod legs differs quite substantially between species. This indicates that other aspects of the Drosophila leg developmental mode are not conserved and are responsible for the morphological differences between the species. The identification of novel leg genes in species other than D. melanogaster can serve as a first step towards a better understanding of the developmental basis of leg diversity in the arthropods. We are studying leg development in the red flour beetle Tribolium castaneum. Tribolium differs from Drosophila in the mode of leg development and in the specific morphology of the legs. Especially in the larva, the legs deviate from the insect typical composition as they lack a separate tibia, lack separate tarsal segments, and have an undivided, claw-shaped pretarsus.

In order to identify genes that play a role in Tribolium leg development we took advantage of enhancer trap lines produced during the GEKU insertional mutagenesis screen conducted in a collaborative effort by four laboratories in Germany and the USA. Details about this screen will be published elsewhere (Trauner et al., manuscript in revision). We have analyzed the enhancer trap lines and have searched specifically for lines expressing the reporter gene [enhanced green fluorescent protein, (EGFP)] in the thoracic legs of Tribolium. This paper reports on the gene zinc finger homeodomain 2 ( $z f h 2)$ that was identified in this process and that was shown to have two striking alternative phenotypes in the legs, linking it to distal development as well as leg segmentation.

\section{Materials and methods}

Characterization of the transposon insertion site

Genomic DNA from imaginal (adult) beetles was isolated by maceration and DNA precipitation. An aliquot of the genomic DNA was digested with Bsp143I, and the fragments were circularized by ligation. Inverse PCR was performed on this template using piggyBac specific primers. The obtained fragments were sequenced directly and the sequence was then compared with the fully sequenced genome of $T$. castaneum by BLAST similarity analysis (Altschul et al. 1997). The sequence obtained from the region flanking the transposon insertion site has been deposited with the EMBL nucleotide database (accession number FN395289).
Molecular cloning, parental RNA interference (RNAi) and whole-mount in situ hybridization

Based on the published genome sequence of $T$. castaneum (Tribolium Genome Sequencing Consortium 2008), a fragment of $z f h 2$ was amplified by PCR from cDNA (prepared from embryos aged 0 to 48 hours) using the primers G03891_fw (TTA GCA TCG GGA AGA CTG GGA) and G03891_rev (GGC TTG GTT GTA GGC CAT GTC). The obtained fragments were cloned and sequenced according to standard methods. Probes for whole-mount in situ hybridization and double-stranded RNA were synthesized based on the fragment isolated with the primers given previously. Whole-mount in situ hybridization was performed according to the published protocol (Prpic et al. 2001). Parental RNAi was performed according to the previously published protocol (Bucher et al. 2002) except that imaginal not pupal beetles were used for injection.

\section{Results}

Analysis of the enhancer trap line Goe-08115

The screen for enhancer trap lines that express EGFP in the legs identified, among other lines, the line Goe-08115. In this line, EGFP protein is detected in embryos shortly before hatching (Fig. 1a) and in larvae (Fig. 1b). EGFP is expressed in the claw and distal end of the tibiotarsus, and in a dorsal spot spanning the trochanter and proximal femur of all thoracic legs. There is also expression in the mandible. In situ hybridization with an EGFP probe revealed that EGFP mRNA is already expressed in elongating embryos (Fig. 1c). The embryonic mRNA pattern is similar to the later protein pattern and consists of a distal domain and a proximal dorsal spot in all thoracic legs and an expression domain in the mandible.

Inverse PCR on genomic DNA prepared from this line and subsequent sequencing identified $324 \mathrm{bp}$ of flanking sequence (see Materials and methods) of the inserted transposon. This sequence matched to the genome sequence of T. castaneum (Tribolium Genome Sequencing Consortium 2008) on chromosome 3 from base pairs 24899583 to 24899906 (refering to genome release 2005 Oct 11) thus identifying the insertion site of the transposon in the genome. Genboree analysis shows a number of predicted genes (GLEAN gene prediction algorithm) in the vicinity of the transposon insertion site. In the area of $100 \mathrm{~kb}$ left and $100 \mathrm{~kb}$ right of the transposon insertion site, 15 genes are predicted by GLEAN (Fig. 1d). We have searched for a Drosophila homolog of each of these predicted genes and have tried to predict the possible function of these genes in order to identify the most promising candidate gene for the 


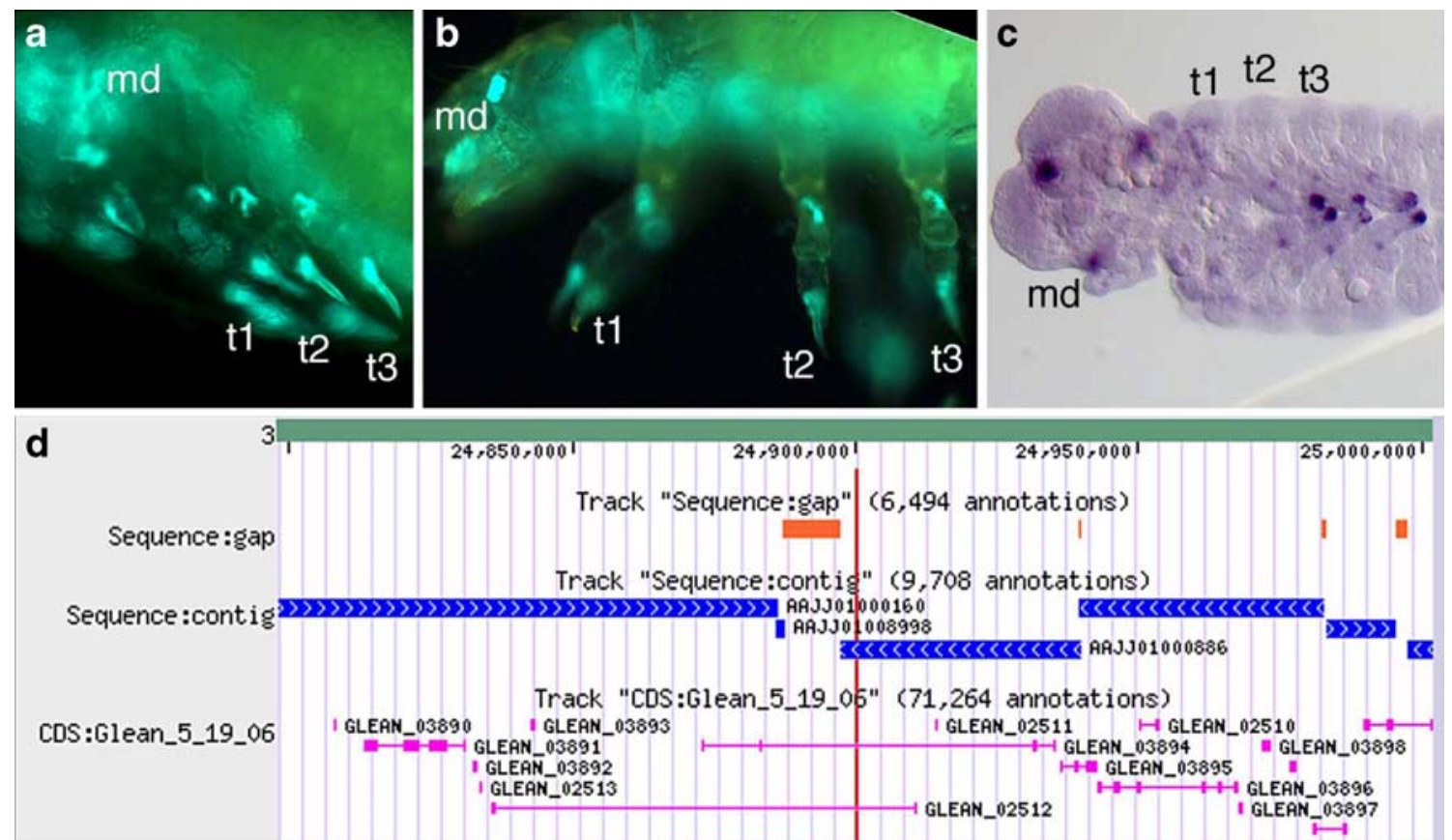

Fig. 1 Localization of the transposon insertion site in the Tribolium line Goe-08115. a, b Detection of EGFP fluorescence (bright green) in late embryonic stages (a) and larvae (b). b Detection of EGFP mRNA (dark purple) in an embryo at the mid-germ band retraction stage. d Screen shot of the genome browser window (at http://www. hgsc.bcm.tmc.edu) identifying annotated details around the transposon insertion site. The frame of the screen shot is centered on the insertion site [i.e. the insertion site is in the middle of the image (red vertical line)]. The green bar at the top gives the base pair count on chromosome three of T. castaneum. The blue bar gives the continuous sequence data information revealing several small gaps (orange color) that have been bridged by genome-mapping data during assembly. The pink bars at the bottom give the locations of the GLEAN predicted genes around the transposon insertion site. The analysis of these GLEANs is shown in Table 1. Abbreviations: $m d$ mandible, $t 1-t 3$ thoracic leg 1 to 3 . The anterior end of the animal is to the left in panels a-c enhancer trap (Table 1). Seven GLEAN predicted genes did not produce a significant match in the GenBank database. This could indicate that these genes are Tribolium-specific or that these predictions are erroneous. The remaining GLEAN-predicted genes correspond to homologous genes in Drosophila, three of which are of unknown function while the remaining genes code for proteins predicted to be cytoplasmic, membrane-bound, or spliceosomal (Table 1). Only a single GLEAN gene, GLEAN_03891, is predicted to encode a nuclear protein containing zinc fingers and homeodomains. BLAST comparison of the sequence of GLEAN_03891 with the Drosophila genome identifies the putative Drosophila ortholog zinc finger homeodomain 2 (zfh2; CG1449). This orthology assessment has also been corroborated by reciprocal BLAST analysis comparing the sequence of CG1449 to the T. castaneum genome.

\section{Expression of $z f h 2$ from $T$. castaneum}

We have then isolated the $z f h 2$ gene from Tribolium and analyzed its embryonic expression pattern. During early germband development $z f h 2$ is expressed in the head lobes and in a separate domain in the growth zone at the posterior end (Fig. 2a, b). In the elongating germ band a strong expression in the entire ventral nervous system appears and the expression in the brain becomes more restricted to several cell groups (Fig. 2c, d; Fig. 3a, b). The developing appendages all express $z f h 2$. In the antenna and the thoracic legs, $z f h 2$ is expressed strongly and homogeneously in the distal half of the appendages (Fig. 3a-c). In the labial and maxillary appendages $z f h 2$ expression is restricted to the distal parts of the palps. The labral buds show a distal spot of zfh 2 expression, and the mandibles express the gene weakly along their ventral edge (Fig. 2c). In the retracting germ band the expression of $z f h 2$ in some tracheal openings appears (Fig. 2e), and in the fully retracted embryo $z f h 2$ is expressed in all tracheal openings, and a stronger expression in the mandibles appears (Fig. 2f). The expression level in the central nervous system increases during germ band elongation. In fully retracted embryos, zfh 2 marks parts of the central nervous system including the two hemispheres of the protocerebrum, segmental ganglia, connectives and commissures. The thoracic legs in fully retracted embryos show several expression domains (Fig. 3d): a proximal ventral stripe (which is weak in the first thoracic segment and stronger in thoracic segment 2 and 3 ) and a proximal dorsal spot, two ring-shaped but punctate medial domains, and a stronger domain in the leg tips. 
Table 1 All genes predicted by the GLEAN algorithm to be located in the vicinity of the transposon insertion site in the line Goe- 08115

\begin{tabular}{lll}
\hline GLEAN number & D. melanogaster ID & Cellular component (www.flybase.org) \\
\hline 02508 & CG3757 & Cytoplasm, extracellular \\
02509 & CG3757 & Cytoplasm, extracellular \\
02510 & CG32597 & Unknown \\
02511 & No significant similarity & - \\
02512 & No significant similarity & - \\
02513 & No significant similarity & - \\
03890 & No significant similarity & - \\
$03891^{\text {a }}$ & CG1449 & Nucleus \\
03892 & No significant similarity & - \\
03893 & No significant similarity & - \\
03894 & CG5339 & Unknown \\
03895 & CG9657 & Membrane \\
03896 & CG32604 & Spliceosome \\
03897 & No significant similarity & - \\
03898 & CG1629 & Unknown \\
\hline
\end{tabular}

The numbers of the GLEAN-predicted genes are given in the first column. The second column gives the orthologous genes, if any, from D. melanogaster [identified by their annotation ID ("CG number")] as inferred from BLAST similarity analysis in the D. melanogaster genome. "No significant similarity" indicates that the GLEAN-predicted gene does not bear similarity to any gene in GenBank. The third column gives the predicted intracellular localization of the gene product as stated in the "cellular component" field on the Flybase Web Service (Drysdale and FlyBase Consortium 2008)

${ }^{\mathrm{a}}$ The GLEAN-predicted gene corresponds to $z f h 2$ and was selected for further study

The dotted appearance of the two medial rings indicates that the expression in these dots might be correlated with peripheral nervous system development probably of sensory organs in the vicinity of the leg joints. The expression pattern of $z f h 2$ thus consists of the enhancer trap pattern plus additional expression domains not detected in the enhancer trap pattern (see Discussion).

RNAi with $z f h 2$ in Tribolium leads to leg development defects

We have then tested the function of the $z f h 2$ gene during Tribolium leg development using RNAi. Surprisingly, two different classes of phenotype were present after $z f h 2$ RNAi. One class comprises legs with fused leg segments (femur and tibiotarsus are fused) and sometimes, ectopic outgrowths (see arrows in Fig. $4 \mathrm{a}-\mathrm{j}$ ). This was the more frequent phenotype $-61 \%$ of all scored larvae $[n=144$ $(100 \%)]$ belonged to this class. We term this phenotype the "femorotarsus" phenotype. The second class of phenotype comprises legs with a reduced and rounded claw but otherwise normal morphology (Fig. 4k-n). This phenotype occurred in $13 \%$ of all scored larvae. We term this phenotype the "smallclaw" phenotype.

The weakest forms of the "femorotarsus" phenotype have all podomeres, but the femur is visibly shorter than wildtype, and the joint between femur and tibiotarsus is not fully formed (Fig. 4a, arrowhead). In other cases, the shortened femur and the tibiotarsus are fully fused (Fig. 4b). In stronger cases, femur and tibiotarsus are not only fused but this large fusion podomere is bent and enlarged ventrally (Fig. 4c, d), and sometimes this enlargement is forming a small outgrowth (Fig. 4e). The formation of ectopic outgrowths can also affect the dorsal side (Fig. $4 \mathrm{f}-\mathrm{h}$ ) or dorsal and ventral side at the same time (Fig. 4j). In rare cases, two separate tips on the same side are present (Fig. 4i) suggesting the presence of two outgrowths but which could also be caused by tumorous growth of a single outgrowth.

The "smallclaw" phenotype is relatively uniform. All leg segments (except for the claw) are present and have their normal length and morphology (Fig. 4k-n). The claw is shortened and is rounded at the end. The length of this malformed claw shows only little variation (Fig. 4k-n).

\section{Discussion}

Comparison of the $z f h 2$ genes of Tribolium and Drosophila

The $z f h 2$ gene has been previously identified in Drosophila. The protein encoded by this gene is unusual in having 16 zinc fingers and three homeodomains in a single protein (Fortini et al. 1991). Conceptual translation of the predicted 
Fig. 2 Expression of $z f h 2$ in Tribolium embryos. a, b In early germ band elongation stages, $z f h 2$ is expressed in the head lobes and in the posterior portion of the embryos.

c Expression in the appendages is restricted to the distal ends in fully elongated embryos.

d, e germ-band retraction, the expression in the central nervous system becomes stronger and more refined. Near the end of germ band retraction $(e)$ the expression in the spiracles (tracheal openings) appears in some segments. (f) In fully retracted embryos, the spiracles of the meso- and metathoracic segment and the abdominal segments express $z f h 2$, as well as the central nervous system and the appendages. Abbreviations: $h l$ head lobes, $g z$ growth zone, lr labrum, $m d$ mandible, $m x$ maxilla. $l b$ labium, $t 1-t 3$ thoracic legs 1 to 3 , cns central nervous system, $s p$ spiracle. Anterior is to the left in all panels
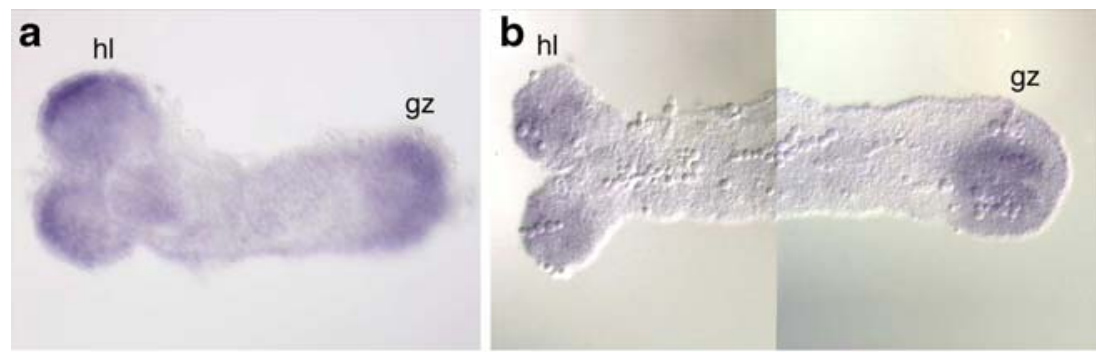

C
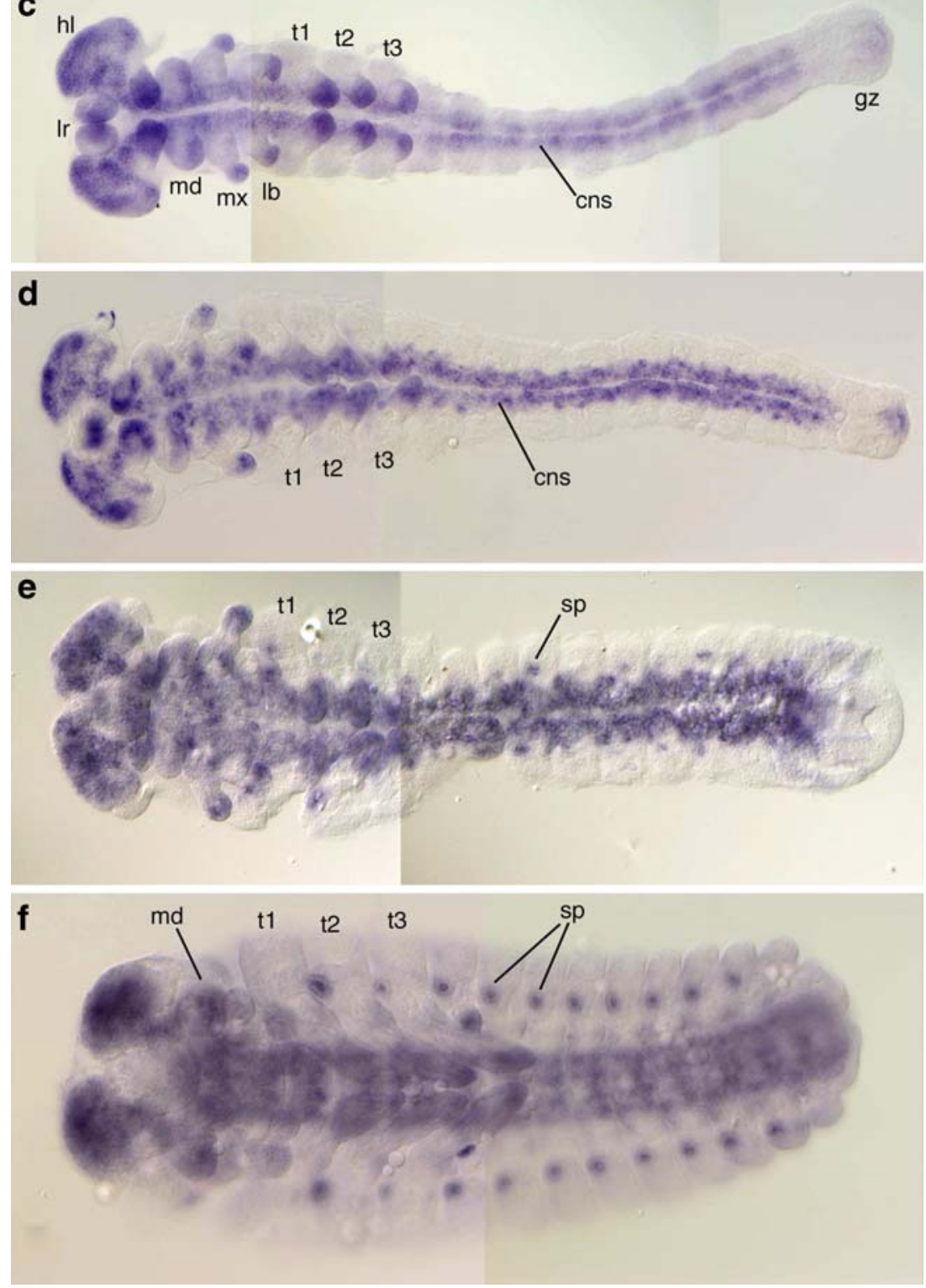

Tribolium zfh2 gene homolog (GLEAN_03891) reveals a similar structure of multiple putative DNA-binding domains. However, Tribolium Zfh2 has 23 zinc fingers and four homeodomains (not shown), and thus is more similar to the mammalian homolog of Zfh2, the AT motifbinding factor 1 (ATBF-1; Morinaga et al. 1991; Hashimoto et al. 1992) that has the identical number of zinc fingers and homeodomains in its longest isoform (Miura et al. 1995; Kawaguchi et al. 2001).

The embryonic expression of $z f h 2$ is very similar in Drosophila (Lai et al. 1991) and Tribolium. Both genes are mainly expressed in the brain and the ventral nerve cord. In addition, Drosophila $z f h 2$ is expressed in the hindgut primordium which might correspond to the posterior 
Fig. 3 Details of $z f h 2$ expression in Tribolium embryos. a, b Gelatine-albumine crosssections through the thorax (a, nearly transversal section) and the head (b, sagittal section) of a mid-elongation stage embryo. Strong expression is seen in the ventral nervous system and in patches in the head lobe and also at the tip of the appendages. c, d Dissected legs showing a single distal domain during germ-band elongation (c) and several additional leg domains in fully retracted stages (d). The two ring domains are denoted by " 1 " and " 2 ", the persisting distal domain is labeled " 3 ". The arrows point to a dorsal and a ventral proximal domain. Please note that the strong expression in the pleuropodium is an artifact seen with any probe at this developmental stage. Abbreviations, see Fig. 2. Additional abbreviations: vns ventral nervous system, $h l$ head lobe, ic tissue of the intercalary segment, an antenna $\mathrm{pl}$, pleuropodium

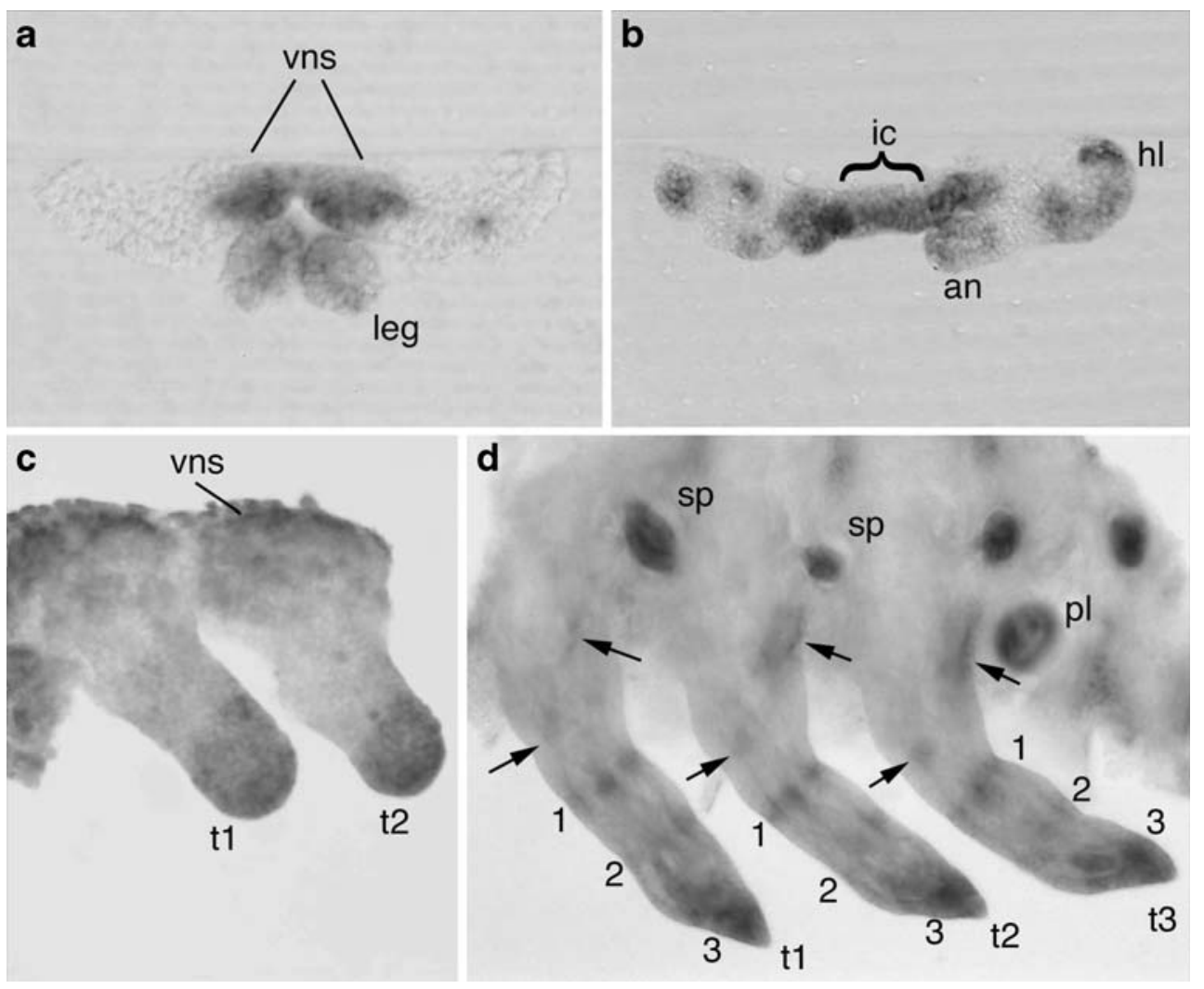

expression in the growth zone in Tribolium. The Drosophila gene is known to be required for nervous system development (Lundell and Hirsh 1992), but a function of $z$ fh 2 in Drosophila leg development has not been reported. However, $z f h 2$ has been demonstrated to play a role in proximal-distal axis formation in the wing disk (Whitworth and Russell 2003). Interestingly, during wing development, $z f h 2$ is regulated downstream of Wingless and Nubbin, which are also known to have roles in distal leg development and leg segmentation, respectively (e.g., Lecuit and Cohen 1997; Rauskolb and Irvine 1999), thus possibly linking $z$ fh 2 to exactly those processes that are disturbed in leg development of Tribolium RNAi phenocopies.

\section{Regulation of $z$ fh 2 in Tribolium}

The enhancer element that has been trapped in the line Goe-08115 obviously is not responsible for producing the entire expression pattern of $z$ fh 2 in Tribolium because the EGFP expression pattern in Goe- 08115 is only a subset of the expression pattern of $z f h 2$ as detected with a $z f h 2$-specific RNA probe. We conclude from this that the enhancer of $z f h 2$ consists of (at least 2, likely more) separate elements for the expression in the nervous system, tracheal openings and appendages. Even the expression domains in the appendages appear to be regulated through different enhancer elements: The enhancer element trapped in line Goe-08115 activates expression in the leg tips as well as in the proximal dorsal spot. Additional domains activated by this enhancer element are in the labrum and in the mandibles. The two ring-shaped domains in the legs, however, must be activated through a different enhancer element. These two separate enhancer elements responsible for activation of $z f h 2$ in the distal and in the ring-shaped domains, respectively, are not only responsible for activating $z$ fh 2 at different locations, but also at different time points. The distal domain element appears to be activated early in development, whereas the ring-shaped domains element is active when the legs are already grown out considerably.

\section{Interpretation of the leg phenotypes}

The two different phenotypes obtained after RNAi require separate interpretation because they appear to be independent from each other and no intermediates between the two have been observed. The "smallclaw" phenotype suggests that the loss of $z f h 2$ leads to difficulties with distal patterning. The $z f h 2$ gene is expressed strongly in the leg tips and the "smallclaw" phenotype suggests that it is necessary there for the normal development of the distalmost podomere, the pretarsus (claw). However, the claw is not lost, only its morphology is disturbed, suggesting that $z f h 2$ is not at the top level in the genetic hierarchy that determines pretarsal fate. Alternatively, the full effect of 




Fig. 4 Spectrum of leg phenotypes obtained after RNAi with $z f h 2$. $\mathbf{a}-\mathbf{j}$ The fusion of femur and tibiotarsus and the formation of ectopic outgrowths characterizes the "femorotarsus" phenotype. The weakest form of this phenotype has a shortened femur, but retains a malformed joint between femur and tibiotarsus (a, arrowhead). Stronger phenotypes have fully fused femur and tibiotarsus, a ventral bend of

loss of zfh2 might be masked by redundancies (e.g., paralogous genes).

The "femorotarsus" phenotype is more difficult to interpret. The $z f h 2$ gene is expressed in two rings in the legs and one of these rings (ring 2 in Fig. 3d) is at the presumptive junction of the femur and the tibiotarsus consistent with a function of $z f h 2$ in the morphogenesis of this joint in the larval leg. Loss of $z f h 2$ is therefore likely to interfere with joint formation and thus might cause the fusion of the two leg segments observed in the "femorotarsus" phenotypes. In Drosophila and the spider Cupiennius salei, proper formation of the leg joints is also necessary for proper growth of the leg segments themselves (de Celis et al. 1998; different severity $\mathbf{b}, \mathbf{c}$ and can have ectopic outgrowths (arrows in $\mathbf{d}-\mathbf{j})$. $\mathbf{k}-\mathbf{n}$ The segmental composition is normal in the "smallclaw" phenotype. The claw abnormally rounded and short $(k, l)$ to very short $(m, n)$. Abbreviations: $c x$ coxa, $t r$ trochanter, $f e$ femur; $t t$ tibiotarsus, $c l$ claw (pretarsus)

Rauskolb and Irvine 1999; Bishop et al. 1999; Prpic and Damen 2009). It is unclear whether podomere growth and joint formation are also coupled in Tribolium, but if so, it could explain the shortening of the femur in the "femorotarsus" phenotypes. These morphological changes indicate a role of $z f h 2$ in leg segmentation. However, the enlargement and the ectopic outgrowths in the "femorotarsus" phenotypes are unexpected and do not seem to be leg segmentation defects. Rather, they appear to be defects with proximal-distal axis formation by inducing supernumerary proximo-distal axes at the fused junction between femur and tibiotarsus. Regeneration experiments in other insect species have suggested that, in the podomeres, a 
gradient of positional information exists that is used to restore the lost appendage parts during regeneration (Bohn 1970a, 1976; Bulliere 1971; Nakamura et al. 2007). However, experimentally joining two leg portions that have different positional values at the joining point results in reversed regeneration or supernumerary proximal-distal axes (Bohn 1970a, 1970b; Mito et al. 2002). This is probably because the joining of incompatible positional values disturbs normal gradient formation that is necessary for the formation of a single proximal-distal axis. The factors involved in these gradients are not all identified, and it is unclear whether these processes that guide regeneration in larval stages play a role in embryonic development as well. However, the reduction of femur size and the fusion of femur and tibiotarsus in the Tribolium "femorotarsus" phenotype might join incompatible positional values in these podomeres and thus lead to disturbances in gradient formation for proximaldistal axis development, which in turn could lead to supernumerary outgrowths in the area of the joining. Undoubtedly, more work is necessary to investigate pattern formation by morphogen gradients in the appendages of Tribolium.

Possible origins of the alternative phenotypes after $z f h 2$ RNAi

An unexpected finding is the existence of the two alternative leg phenotypes "femorotarsus" and "smallclaw" without any intermediate or combined phenotypes. These phenotypes could be caused by small differences in the effectivity of the RNAi in the respective appendage. Two alternative results depending on the absolute concentrations or the ratio of two factors are known from the morphogen regulation of target genes (dachshund, Distal-less) during Drosophila leg development (Estella and Mann, 2008). Zfh2 may regulate the expression of one or several target genes that act as morphogens. Alternatively, the target gene(s) may have a composite regulatory region in which several elements compete for Zfh2 binding to either repress or activate the target gene, with different outcomes of the competition in claw cells and in joint cells, respectively. Interfering with $z f h 2$ expression might disrupt this sensitive competition system in different ways in the different cell types and at different RNAi efficiencies.

Another possibility is that $z f h 2$ RNAi interferes directly with allometric leg growth. This primary defect could lead to a secondary disturbance of the leg patterning network, for example because there is not enough space for the regular number of joints at the required distances. The attempts of the regulatory network to compensate for the defect could lead to two different outcomes because they are the only two stable alternatives to the wildtype situation.
Finally, we note that recent work has shown that the translation of Drosophila $z f h 2$ is regulated by the micro-RNA $m i R-276 a, b$ (Stark et al. 2005) and thus by an RNAi-related mechanism. We think that it is possible, that RNAi interferes with miRNA regulation of $z f h 2$ to lead to the two observed alternative outcomes.

The occurrence of two alternative phenotypes after RNAi is, to our knowledge, unprecedented. Clearly, the possible explanations given in the discussion above are just attempts to provide first ideas about how this phenomenon could be brought about. More work is thus required to investigate further the possible miRNA regulation of Tribolium zfh 2 and the interactions and regulatory targets of this multidomain protein.

Acknowledgements We thank Matthias Pechmann for helpful discussions and Nico Posnien for support with the Tribolium work. We also thank Elke Küster for the stock-keeping of the GEKU lines. This work has been funded by the Deutsche Forschungsgemeinschaft [grants PR1109/1-1 (NMP), BU-1443/3-1 (GB)] and the United States Department of Agriculture (USDA) grant number CSREES_NRI 2004-35604-14250 (EAW).

Open Access This article is distributed under the terms of the Creative Commons Attribution Noncommercial License which permits any noncommercial use, distribution, and reproduction in any medium, provided the original author(s) and source are credited.

\section{References}

Abzhanov A, Kaufman TC (2000) Homologs of Drosophila appendage genes in the patterning of arthropod limbs. Dev Biol 227:683-689

Altschul SF, Madden TL, Schäffer AA, Zhang J, Zhang Z, Miller W, Lipman DJ (1997) Gapped BLAST and PSI-BLAST: a new generation of protein database search programs. Nucleic Acids Res 25:3389-3402

Beermann A, Jay DG, Beeman RW, Hülskamp M, Tautz D, Jürgens G (2001) The short antennae gene of Tribolium is required for limb development and encodes the orthologue of the Drosophila Distal-less protein. Development 128:287-297

Bishop SA, Klein T, Martinez Arias A, Couso JP (1999) Composite signalling from Serrate and Delta establishes leg segments in Drosophila through Notch. Development 126:2993-3003

Bohn H (1970a) Interkalare Regeneration und segmentale Gradienten bei den Extremitäten von Leucophaea-Larven (Blattaria). I. Femur und Tibia. Roux Arch 165:303-341

Bohn H (1970b) Interkalare Regeneration und segmentale Gradienten bei den Extremitäten von Leucophaea-Larven (Blattaria). II. Coxa und Trochanter. Dev Biol 23:355-379

Bohn H (1976) Tissue interactions in the regenerating cockroach leg. In: Lawrence PA (ed) Insect development. Blackwell Scientific Publications, Oxford, pp 170-185

Bucher G, Scholten J, Klingler M (2002) Parental RNAi in Tribolium (Coleoptera). Curr Biol 12:R85-R86

Bulliere D (1971) Utilisation de la regeneration intercalaire pour l'etude de la determination cellulaire au cours de la morphogenese chez Blabera craniifer (Insecte Dyctioptere). Dev Biol 25:672-709

Cohen B, Simcox AA, Cohen SM (1993) Allocation of the thoracic imaginal primordia in the Drosophila embryo. Development 117:597-608 
de Celis JF, Tyler DM, de Celis J, Bray SJ (1998) Notch signalling mediates segmentation of the Drosophila leg. Development 125:4617-4626

Drysdale R, FlyBase Consortium (2008) FlyBase: a database for the Drosophila research community. Methods Mol Biol 420:45-59

Estella C, Mann RS (2008) Logic of Wg and Dpp induction of distal and medial fates in the Drosophila leg. Development 135:627-636

Fortini ME, Lai ZC, Rubin GM (1991) The Drosophila $z f h-1$ and $z f h-2$ genes encode novel proteins containing both zinc-finger and homeodomain motifs. Mech Dev 34:113-122

Goto S, Hayashi S (1997) Specification of the embryonic limb primordium by graded activity of Decapentaplegic. Development 124:125-132

Hashimoto T, Nakano Y, Morinaga T, Tamaoki T (1992) A new family of homeobox genes encoding multiple homeodomain and zinc finger motifs. Mech Dev 39:125-126

Kawaguchi M, Miura Y, Ido A, Morinaga T, Sakata N, Oya T, Hashimoto-Tamaoki T, Sasahara M, Koizumi F, Tamaoki T (2001) DNA/RNA-dependent ATPase activity is associated with ATBF1, a multiple homeodomain-zinc finger protein. Biochim Biophys Acta 1550:164-174

Kubota K, Goto S, Eto K, Hayashi S (2000) EGF receptor attenuates Dpp signaling and helps to distinguish the wing and leg cell fates in Drosophila. Development 127:3769-3776

Kubota K, Goto S, Hayashi S (2003) The role of Wg signaling in the patterning of embryonic leg primordium in Drosophila. Dev Biol 257:117-126

Lai Z, Fortini ME, Rubin GM (1991) The embryonic expression patterns of $z f h-1$ and $z f h-2$, two Drosophila genes encoding novel zinc-finger homeodomain proteins. Mech Dev 34:123-134

Lecuit T, Cohen SM (1997) Proximal-distal axis formation in the Drosophila leg. Nature 388:139-145

Lundell MJ, Hirsh J (1992) The $z f h-2$ gene product is a potential regulator of neuron-specific DOPA decarboxylase gene expression in Drosophila. Dev Biol 154:84-94

Mito T, Inoue Y, Kimura S, Miyawaki K, Niwa N, Shinmyo Y, Ohuchi $\mathrm{H}$, Noji S (2002) Involvement of hedgehog, wingless, and dpp in the initiation of proximodistal axis formation during the regeneration of insect legs, a verification of the boundary model. Mech Dev 114:27-35

Miura Y, Tam T, Ido A, Morinaga T, Miki T, Hashimoto T, Tamaoki T (1995) Cloning and characterization of an ATBF1 isoform that expresses in a neuronal differentiation-dependent manner. J Biol Chem 270:26840-26848

Morinaga T, Yasuda H, Hashimoto T, Higashio K, Tamaoki T (1991) A human alpha-fetoprotein enhancer-binding protein, ATBF1, contains four homeodomains and seventeen zinc fingers. Mol Cell Biol 11:6041-6049

Nakamura T, Mito T, Tanaka Y, Bando T, Ohuchi H, Noji S (2007) Involvement of canonical Wnt/Wingless signaling in the determination of the positional values within the leg segment of the cricket Gryllus bimaculatus. Dev Growth Differ 49:79-88

Prpic NM, Wigand B, Damen WGM, Klingler M (2001) Expression of dachshund in wild-type and Distal-less mutant Tribolium corroborates serial homologies in insect appendages. Dev Genes Evol 211:467-477

Prpic NM, Janssen R, Wigand B, Klingler M, Damen WGM (2003) Gene expression in spider appendages reveals reversal of exd/hth spatial specificity, altered leg gap gene dynamics, and suggests divergent distal morphogen signaling. Dev Biol 264:119-140

Prpic NM, Damen WGM (2009) Notch-mediated segmentation of the appendages is a molecular phylotypic trait of the arthropods. Dev Biol 326:262-271

Rauskolb C, Irvine KD (1999) Notch-mediated segmentation and growth control of the Drosophila leg. Dev Biol 210:339-350

Schoppmeier M, Damen WGM (2001) Double-stranded RNA interference in the spider Cupiennius salei: the role of Distal-less is evolutionarily conserved in arthropod appendage formation. Dev Genes Evol 211:76-82

Stark A, Brennecke J, Bushati N, Russell RB, Cohen SM (2005) Animal MicroRNAs confer robustness to gene expression and have a significant impact on 3'UTR evolution. Cell 123:11331146

Trauner J. Schinko J, Lorenzen MD, Shippy TD, Wimmer EA, Beeman RW, Klingler M, Bucher G, Brown SJ (in revision) Large-scale insertional mutagenesis in the red flour beetle Tribolium castaneum identifies embryonic lethal mutations and enhancer traps

Tribolium Genome Sequencing Consortium (2008) The genome of the model beetle and pest Tribolium castaneum. Nature 452:949955

Whitworth AJ, Russell S (2003) Temporally dynamic response to Wingless directs the sequential elaboration of the proximodistal axis of the Drosophila wing. Dev Biol 254:277-288 